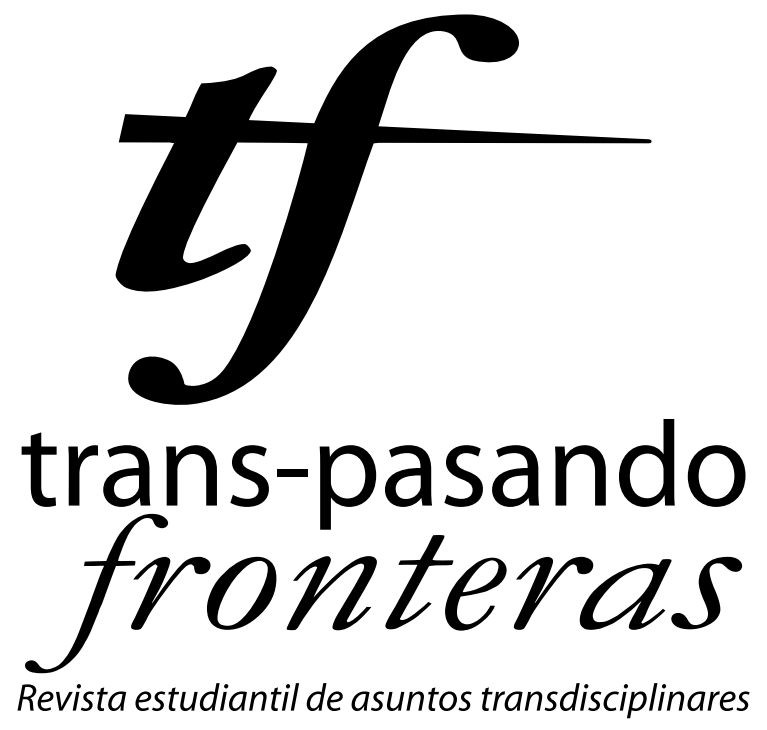

Una publicación de

Facultad de Derecho y Ciencias Sociales

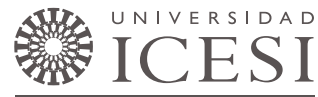




\title{
Hugo Chávez y la crisis de representación político-partidaria en Venezuela
}

\author{
Alejandra Arias García* \\ (pdg.alearias@gmail.com)
}

Artículo de investigación científica y tecnológica recibido el 28/06/2012 y aprobado el 17/10/2012.

Cómo citar este artículo:

ARIAS GARCÍA. Alejandra (2012). "Hugo Chávez y la crisis de representación político-partidaria en Venezuela”. En: Trans-pasando Fronteras, Núm. 2, pp. 65-83. Cali, Colombia: Centro de Estudios Interdisciplinarios, Jurídicos, Sociales y Humanistas (CIES), Universidad Icesi.

\begin{abstract}
Resumen
Este artículo analiza la actual situación política de Venezuela como una consecuencia directa del colapso del sistema de partidos, cuyos actores tradicionales fueron el AD y el COPEI. Presenta el régimen populista de Hugo Chávez como un "autoritarismo competitivo", en la medida en que la crisis de representación no pasa necesariamente por la contienda electoral, sino que se basa en la cooptación del ejecutivo de otras arenas de poder como la legislativa, la judicial y la regulación de los medios de comunicación.
\end{abstract}

\footnotetext{
* Estudiante de ciclo básico de la Maestría en Estudios Latinoamericanos de la Universidad Nacional de Cuyo, Argentina. Antropóloga y politóloga graduada de la Universidad Icesi en el año 2012. Sus áreas de interés son: migración por conflicto armado (refugio político y desplazamiento forzado), el sistema-mundo contemporáneo y la posición latinoamericana y políticas sociales en América Latina. Entre sus ponencias se encuentran: "El desplazamiento forzado transfronterizo de colombianos a Ecuador. Un análisis del refugio político como resultado de la dependencia político-militar de Colombia hacia Estados Unidos”. Presentada en 2012, en las I Jornadas de Estudios de América Latina y el Caribe, en la Universidad de Buenos Aires, Argentina.
} 


\section{Palabras claves:}

Hugo Chávez, populismo, autoritarismo competitivo, sistema de partidos, crisis de representación política.

\section{Introducción}

El mundo de la post-guerra fría se caracterizó por un número considerablemente nuevo de regímenes que empezaban su transición hacia la democracia. Otros se quedaron en sistemas híbridos que mezclaban regímenes autoritarios con democráticos. Sin embargo, existe también un fenómeno considerable de casos que han tenido el proceso contrario: pasaron de ser regímenes democráticos a no democráticos, lo que deja en evidencia una clara crisis de representación política, en tanto grupos que antes podían competir satisfactoriamente en la arena política quedaron excluidos de la misma (Levitsky y Way, 2004).

Venezuela es uno de estos casos en los que la democracia queda actualmente en entredicho, convirtiéndose en lo que Levitsky y Way (2004) denominan un autoritarismo competitivo. Se caracteriza por la realización de elecciones competitivas sin que necesariamente esto implique la existencia de un régimen democrático. Eso hace que se constituya en un caso teóricamente importante para ser evaluado. En términos empíricos, la cuestión no es menos trascendente. Venezuela se ha distinguido, desde la llegada de Hugo Chávez al poder, por la represión de la oposición como forma de gobierno. Esta represión se ha visto reflejada en fenómenos como la disminución de la información, del debate público y un poder legislativo controlado por el ejecutivo, desequilibrando el principio democrático de pesos y contrapesos (Corrales, 2010).

En este artículo se aborda la crisis de representación política desde el colapso del sistema de partidos. Sostendrá la idea de que la caída del bipartidismo venezolano, considerado como uno de los más estables de Latinoamérica en la década de los 80 , se convirtió en una de las causas fundamentales para la instauración del autoritarismo competitivo de Hugo Chávez, entre 1998 y 2012.

Se retomarán los postulados teóricos propuestos por el institucionalismo sociológico, que expone que "los cambios institucionales son producto de un cambio de los actores, (y, particularmente de una modificación en el ambiente), en la socialización y en la propia cultura (tradiciones y valores) [...] por consiguiente, tres variables integran el planteamiento del institucionalismo sociológico: las instituciones o agencias, el medio 
ambiente, los procesos de socialización y, naturalmente, el individuo” (Rivas, 2000:71). El institucionalismo sociológico ayuda a explicar el porqué del colapso del sistema bipartidista y sus consecuencias: (i) un cambio de actores, donde los partidos políticos tradicionales perdieron legitimidad como vehículos de los intereses ciudadanos y dieron cabida a un presidencialismo personalista; y (ii) un ambiente de insatisfacción por las ineficientes respuestas a las demandas sociales. Estas condiciones permitieron que Hugo Chávez se instaurara legítimamente como alternativa a dicha insatisfacción. Una vez ocupó el poder ejecutivo, dio pie al cambio de las instituciones formales, como la constitución política y la toma de decisiones por medio de la democracia plebiscitaria. Esto le brindó una capacidad mayor de acción desde el ejecutivo desequilibrando el sistema de equilibrio de pesos y contrapesos (Rivas, 2000 y Corrales, 2010).

El artículo, en un primer momento, explicará cómo se produjo el colapso del sistema de partidos venezolano. Seguidamente, presentará una breve historia de la vida política de Hugo Chávez y caracterizará el fenómeno del populismo chavista. En un tercer instante, analizará el papel de los partidos políticos oficialistas. Finalizará exponiendo los cambios institucionales que llevaron a que Venezuela se considere, actualmente, un autoritarismo competitivo.

\section{Del multipartidismo moderado al bipartidismo: antecedentes del colapso}

El sistema de partidos venezolano fue durante su historia democrática un sistema bipartidista conformado por el partido Acción Democrática (AD) y el Partido Demócrata Cristiano (COPEI). Para 1935, luego de la dictadura de Juan Vicente Gómez, Venezuela entró a una transición democrática bastante inestable durante la primera mitad del siglo XX. Durante dicho tiempo, parecía que su sistema de partidos iba a tender hacia un pluralismo moderado con la existencia de cuatro partidos, dos además de los ya nombrados: el Partido Comunista Venezolano (PCV) y la Unión Republicana Democrática (URD). Sin embargo, el AD tomó una fuerza importante como organización partidaria, de tal manera que uno de sus gobiernos fue objeto de un golpe de Estado militar:

Tres años de gobierno revolucionario (1945-1948) llevaron al partido AD a una posición dominante. Pero el uso del poder gubernamental por parte de AD para limitar el crecimiento de los partidos URD, Copei y PCV los alienó del flamante 
régimen democrático representativo que $\mathrm{AD}$ intentaba construir. Por consiguiente, URD, Copei y PCV se echaron a un lado en noviembre de 1948, cuando los militares derrocaron al gobierno electo del presidente Rómulo Gallegos (AD). Los militares, sin embargo, no tenían ninguna intención de permitir que URD, Copei o el PCV gobernara. Este hecho hizo que los tres partidos se unieron al AD para reformar un movimiento clandestino que derrocó al gobierno militar del General Marco Pérez Jiménez el 23 de enero de 1958” (Dietz y Myers, 2002, pág. 10).

A partir de la alianza entre los partidos AD, COPEI, URD y PCV se creó el Pacto del punto fijo, acuerdo que pretendía que éstos se repartieran el poder de tal manera que la democracia no se viera nuevamente amenazada. Tal acuerdo suponía una competencia real por el poder y la implementación del multipartidismo. Empero, el ambiente electoral y el contexto político marcó una ruta distinta. Primero, el URD se convirtió en un partido insignificante por su falta de adeptos en la arena electoral y, segundo, el PCV entró en un conflicto ideológico en tanto unos querían apoyar la causa de Fidel, mientras otros, los más jóvenes, veían en la Unión Soviética su ejemplo a seguir. Así que dividió el partido en dos y llevó a que la izquierda no llegara a tener una posibilidad real de acceder al poder (Dietz y Myers, 2002).

En congruencia con todo lo anterior y en la medida en que en la contienda electoral solo tuvieron primacía el AD y el COPEI, se habla de Venezuela como un sistema bipartidista.

\section{Desplome del sistema bipartidista venezolano}

Un colapso del sistema de partidos comienza a gestarse a partir de cierto sentimiento de desconfianza del electorado, lo que brinda un espacio para que políticos con orientación anti-élite puedan acceder al poder o, como en otras ocasiones ha pasado, que se produzcan golpes de estado por parte de facciones militares. Cuando se habla de que un sistema de partidos ha colapsado, se hace referencia a dos ideas. Primero, que ha existido un proceso de implosión repentina, en vez de uno gradual, logrando su culminación absoluta en un lapso relativamente corto. Segundo, una falta de adaptabilidad del mismo, en tanto a que no logra moldearse a contextos sociales y políticos inestables. En otras palabras, los sistemas de partido no colapsan de manera fortuita. Siempre existen razones para que se presente su derrumbe: un sistema demasiado rígido que no permita la entrada de nuevos integrantes o candidatos, entre otro tipo de razones. Siguiendo esta premisa, el colapso 
solo se genera cuando existe un divorcio directo entre los representantes y los ciudadanos a los que representan, es decir, a los electores (Dietz y Myers, 2002).

El AD y el COPEI eran partidos políticos representativos y tenían gran arraigo en la sociedad, en tanto que se relacionaban de manera directa con diversos grupos de la sociedad civil, "lo que fomentó la integración social y la participación cívica de los sectores excluidos" (Tanaka, 2008:91). A pesar de lo anterior, la década de los 80 marcó un cambio estructural en la composición del sistema de partidos de Venezuela. La desafección de los ciudadanos hacia sus partidos estuvo basada en el desempeño de la economía y, primordialmente, en las luchas internas y los procesos de fracturación intrapartidaria.

Los conflictos internos en ambos partidos debieron sus causas a la extrema rigidez y severa jerarquía institucional. Se limitó su capacidad de reacción frente a las distintas reformas políticas que indicaban un proceso de descentralización, como la elección popular directa de gobernadores y alcaldes, que pretendía "abrir un sistema político percibido como herméticamente cerrado (caracterizado por una "partidocracia"), renovar el liderazgo y reducir el control de los caciques partidarios" (Tanaka, 2008:105). En la medida en que la efectividad de las reformas no fue la esperada, se aumentó el conflicto interno de los partidos que, junto a la crisis económica, llevó a la deslegitimación de los mismos.

Entender el fenómeno del conflicto intrapartidario de una mejor forma requiere hacer un examen de los dos partidos de manera separada. Por un lado, el AD era considerado "una estructura burocratizada y en muchos casos corrupta, habituada a las relaciones clientelistas y corporativas y a los favores financieros" (Tanaka, 2008: 106). Lo anterior sumado a las reformas, causó que en 1988 no fuera un candidato oficialista el que ganara las elecciones como representante del partido sino Carlos Andrés Pérez que había sido presidente diez años antes. Esto acarreó tensión dentro del partido en la medida en que al ganar de nuevo la presidencia, decidió crear un equipo de gobierno independiente que le permitiera ejercer un mandato con cierto grado de discrecionalidad de las decisiones del partido al que pertenecía. Sus políticas estuvieron relacionadas con las reformas neoliberales y sus copartidarios se sintieron aislados de la toma de decisiones oficiales. Además, la mala situación económica venezolana quedó ejemplarizada en indicadores como el déficit del sector público, las alzas del precio del petróleo y los niveles de hiperinflación, que llevaron a que la base social del $\mathrm{AD}$ (la Confederación Sindical de Trabajadores) se alejara del partido, lo que constituyó un alto costo social. 
La desarticulación de Pérez con el AD es una clara muestra de la implosión que estaba sufriendo el partido. Su legitimidad se soslayó, ya de forma definitiva, con las reacciones que tuvo en la sociedad su programa de reformas, pues tuvo oposición desde su partido y otros sectores insatisfechos. No solo el gobierno enfrentó las manifestaciones de masas y dos intentos de golpe de estado, sino que también se vio debilitado por todas las rencillas internas que el AD tuvo con su gestión. Todas estas querellas, condujo a que los afiliados abandonaran el partido en las elecciones de 1993 y en 1998 decidiera no postular a nadie por no contar con el apoyo de los venezolanos en las encuestas de opinión (Levitsky y Burguess, 2003).

La situación del COPEI no fue distinta en su comportamiento interno, caracterizado por un nivel de institucionalización bajo. El partido no apoyó al que había sido su fundador y presidente, Rafael Caldera, en las elecciones de 1988, lo que lo llevó a su separación del partido para las elecciones de 1993 y su postulación por el grupo Convergencia Nacional. El hecho significó el termino de la alternancia de poder, en la medida en que Caldera introdujo un discurso populista que atrajo una ola de simpatizantes golpistas que estaban en contra de las reformas neoliberales de Pérez. Así, Caldera se llevó gran parte del colchón electoral del COPEI, lo que fraccionó y debilitó al partido, hasta el punto de no presentar candidato a la presidencia para las elecciones de 1998.

Todo lo anterior refleja la transformación del sistema de partidos venezolano y evidencia la deslegitimación del mismo, en la medida en que ni los partidos tradicionales ni los nuevos partidos pudieron satisfacer las expectativas del electorado. La llegada de Chávez no correspondió solo al descontento generalizado por la situación económica, sino también a la desestructuración de los partidos tradicionales que no permitió tener candidaturas fuertes para hacer contrapeso.

\section{El chavismo como fenómeno populista}

Hablar de chavismo como populismo requiere definir qué se entiende por el último término. Se considera "populismo" a un tipo de liderazgo político personalista, cuyo líder cuenta con una imagen carismática. Funciona a partir de una coalición electoral heterogénea, que busca la adhesión de los sectores históricamente excluidos de la vida política nacional. Por último, hace referencia a una estrategia política de movilización donde se 
prescinden de los partidos políticos como modelos institucionalizados de representación; se apela a la alternativa de la relación directa del líder con el pueblo (De la Torre, 2000).

A partir de lo anterior, es necesario analizar la historia de Chávez en la esfera pública venezolana desde antes de que accediera al poder político, solo así es posible entender su actual régimen como un fenómeno populista. En 1970, oficiales del rango medio encabezados por Hugo Chávez se comprometen con el activismo político y los cambios radicales en la forma de concebir las instituciones militares. Esto se puede observar a través de tres procesos: (i) preparar a los militares en las universidades como una alternativa a las instituciones militares, lo que causa una alta polarización académica, (ii) las guerrillas venezolanas, que se encontraban desde la década de los 60, tratan políticamente que los militares entren a la causa revolucionaria. Por último, (iii) la radicalización política queda materializada en un movimiento clandestino que emerge con el nombre de Movimiento Bolivariano Revolucionario 200 (MBR-200) creado por Hugo Chávez en 1983, y que pretendía cambiar la desigualdad y la corrupción del sistema político venezolano (Hawkins, 2010).

Una fecha determinante en la historia de Hugo Chávez es febrero de 1989, durante el gobierno de Carlos Pérez. Mes en que se lleva a cabo el denominado Caracazo, un motín masivo que surge en respuesta al incremento de los precios de la gasolina, producto del ajuste y las reformas económicas que Pérez aplicó. Lo anterior lleva a que se creen fuertes represiones contra los sectores más desfavorecidos. El inicio de 1992 es un momento emblemático para este movimiento ya que existe una opinión pública desencantada del sistema que se encontraba vigente, lo que genera posiciones que favorecen al ejército. Chávez el 4 de febrero de 1992 lleva a cabo, junto a los otros militares pertenecientes MBR-200, el fallido golpe de Estado.

La imagen de Chávez se empieza a mediatizar desde su último evento. A partir de ahí, se le conoce como el representante del carisma, la juventud, la voluntad de hacer el cambio a través del manejo de un lenguaje sencillo y de mucho impacto. Se le sentencia a dos años de cárcel. Después de esto, el gobierno de turno lo llama a participar de la vida política pero pacíficamente. Chávez y otros militares del núcleo radical rechazan la oferta y empiezan a difundir, por su lado, ideas de una revolución democrática. Dado que el MBR-200 tiene que disolverse después del golpe de Estado, en 1997 se firma una carta para fundar el Movimiento de la V República (MVR) y postula a Chávez como su candidato presidencial, siendo elegido para este cargo en 1998 (Hawkins, 2010). 
Toda esa coyuntura además del colapso del sistema de partidos que se trató en el punto anterior, ayuda a entender la manera en la que el chavismo se forja como fenómeno populista. Hawkins (2010) plantea dos criterios fundamentales para definir un populismo: en primer lugar, la modalidad carismática como se vinculan votantes y líder político; $\mathrm{y}$, en un segundo momento, la utilización de un discurso democrático basado en la idea de la voluntad popular, donde el "pueblo" pasa a ser más importante que la "élite". Es de este modo como el populismo articula una relación de corte carismática entre el líder y el pueblo que se genera bajo una modalidad particular de intercambio entre votantes y políticos: intercambio de votos y promesas de un cambio radical por parte de una persona con habilidades y carácter extraordinarios, incluso, percibido por muchos como cualidades casi divinas. Aquí, la imagen del líder importa más que el contenido de las promesas que hace. Por lo general, este tipo de liderazgo político surge en períodos de crisis institucional, momento que aprovecha para reducir la incertidumbre, por un lado, y evitar un comportamiento oportunista por parte de otros políticos, por el otro, a través de su discurso político. El populismo se ha caracterizado por prescindir de los partidos políticos como puente entre la política y la ciudadanía. En tanto el valor recae completamente sobre la particularidad de una persona, los partidos se pueden convertir, al contrario, en un obstáculo para la consecución de sus intereses individuales. Por consiguiente, la democracia directa o plebiscitaria se convierte en la mejor forma de obtener un poder legitimado en la figura del "pueblo" y el líder populista sólo como aquel ejecutor de esas decisiones (Hawkins, 2010).

\section{Del MVR al PSUV: la falta de institucionalización de los partidos chavistas.}

Reconocer que las elecciones siguen siendo la forma política utilizada en Venezuela, hace preciso mostrar la falta de institucionalización de los partidos chavistas. Para esto, es necesario analizar las dos principales fuerzas políticas que han servido de vehículos de ascenso y estabilización de Hugo Chávez en el poder: el MVR y el PSUV ${ }^{1}$.

$1 \quad$ Es preciso decir que el MVR existió hasta el 2007, momento en el que se integró al Partido Socialista Unido de Venezuela -PUSV-. Al presente, el PSUV representa la base electoral más fuerte que apoya la llamada "Revolución Bolivariana". 
La principal característica del chavismo como fenómeno populista es la vinculación carismática del líder con el pueblo y la débil institucionalización del partido o movimiento político que lo representa, dependiendo del momento político, para lograr instaurar su posición. El MVR o el PUSV no constituyen partidos políticos sólidos, primordialmente porque no cumplen ninguna de las condiciones necesarias para que se los considere fuertemente institucionalizados, en términos de las raíces en la sociedad, legitimación del proceso electoral, la fortaleza organizativa y la volatilidad electoral.

Entender el arraigo en la sociedad de un partido político, implica una posición efectiva que marque una identidad de partido, y que sea interiorizada de manera tal por la sociedad que una ruptura de la organización con esa identidad pueda representar un fracaso en la arena electoral (Mainwaring y Scully, 2004). La personalización de la política venezolana en la figura de Chávez, implica que ni el MVR, para sus primeros años de gestión, ni el PUSV, para los últimos, se hayan constituido en partidos políticos con líneas programáticas claras e identidades fácilmente referenciales.

Esto queda demostrado en la indefinición de sus reglas de juego, pues son variables a las consideraciones de Chávez como líder. Lo anterior lleva a que los partidos o movimientos que representan a Chávez no puedan generar al interior de sus organizaciones posturas políticas propias o discrepantes de las autoridades partidarias, respuestas a crisis, o permitan el acceso efectivo de nuevos líderes (Agulló, 2009).

La falta de institucionalización permite crear otro tipo de organizaciones de base paralelas a la representación partidaria, que se convierten en reguladoras de las actuaciones de los partidos. Los "círculos bolivarianos" son muestras de este fenómeno. Se crean para hacerle frente a tendencias faccionistas que empiezan a extenderse y se presentan como una fórmula para fomentar la autoayuda y el desarrollo del pueblo, a nivel local y desde abajo. Los círculos, además, también sufren de una baja institucionalización en tanto no poseen capacidad de acción propia más allá de la que Chávez mismo les proporciona. Poseen objetivos demasiado diversos y falta de organización. Chávez decide crear, desde su posición de poder, este tipo de organizaciones que, aunque son partes del partido, se caracterizan por actuar de forma independiente del mismo y, en muchas ocasiones, contradicen las decisiones del mismo (Hawkins, 2007). 
Es difícil dilucidar si existe o no una legitimación del proceso electoral, si se la entiende como el reconocimiento de otros actores y partidos de un proceso de elecciones aceptados (Mainwaring y Scully, 2004). El AD y el COPEI, los dos partidos que desde sus procesos de implosión llevaron al colapso del sistema, aludieron en muchas ocasiones a irregularidades de los procesos electorales, una vez Chávez subió a ocupar el primer cargo del ejecutivo. No obstante, Chávez siempre ha permitido la evaluación de los procesos electorales por parte de observadores internacionales de la OEA, que han llegado siempre a la conclusión de que las elecciones se generaron en condiciones limpias y justas. Esto desincentivó a tal punto a la oposición que en las elecciones legislativas de 2005 se retiraron la mayoría de sus candidatos (Gamus, 2006).

La fortaleza organizativa es el problema más grave que enfrentó la institucionalización de los partidos que representan a Chávez. Se refiere al grado de autonomía partidaria e involucra la existencia de estructuras firmemente establecidas, territorialmente extensas, organizadas y con recursos propios (Mainwaring y Scully, 2004).

Antes de su disolución, el MVR tenía muy poca autonomía, fue una organización básica; con capacidad de coalición floja de distintos partidos e ideologías. Fue un núcleo cohesionador que Chávez poseía para manejar el poder legislativo. No tenía ni identidad ni reglas sólidas: solo existía para ejecutar los deseos políticos de Chávez. Por último, a pesar de que en su visión se establecía un funcionamiento interno democrático, en realidad todo se manejaba desde el nivel nacional, sin llamar a consultas populares (por ejemplo selección de candidatos para elecciones) (Hawkins, 2007). Todo lo anterior lleva a intuir que el MVR nunca fue un partido realmente autónomo. Al contrario, su disolución dependió explícitamente del deseo de Hugo Chávez de integrarlo al Partido Socialista Unido de Venezuela (PSUV).

El PSUV, por su parte, ha sido cuestionado desde su creación por la instauración de un "Tribunal disciplinario" que pretende "sancionar" a aquellos militantes que incumplan con sus deberes formales dentro de la tolda o realicen acciones que acarreen algún perjuicio contra el PSUV. El cuestionamiento primordial a esta instancia, es que se estableció antes de que se tuvieran claras cuáles serían las doctrinas del partido, sus criterios, principios y posturas ideológicas. Es imposible pensarlo como "democrático" cuando, desde su inicio, se restringe el debate y la disidencia (Lander, 2007). 
Por último, la volatilidad electoral, hace referencia a la estabilidad en las reglas (Mainwaring y Scully, 2004). El cambio de las reglas de juego empezó con la instauración de la Constitución Bolivariana de Venezuela y siguió con la repetida utilización del plebiscito como forma de gobierno directo. Al día de hoy, se han presentado más de siete referendos; los más importantes, en términos electorales, son: los referendos de 1998 que llevaron a que se cambiaran la Asamblea Nacional de bicameral a unicameral. Eso llevó a que se llamara a elecciones legislativas extraordinarias donde la oposición quedó opacada. De la misma manera, en el 2009 planteó otro referendo en el que pedía permitir la postulación a cualquier cargo de elección popular de manera continua. Esto, claro, deseaba hacerlo para poder perpetuarse en el poder más allá de lo que la constitución misma lo permitía. Chávez ha sabido manejar a su favor el cambio de las reglas -instituciones- para poder instaurar una forma de gobierno que le dé más capacidad de acción (Rivas, 2000).

\section{Debilidad institucional del sistema de partidos y crisis de representación}

El principal problema del colapso del sistema de partidos recae en la representación política misma. Cuando el electorado no rechaza uno de los partidos políticos sino el sistema, las repercusiones recaen sobre la democracia (Dietz y Myers, 2002). Una vez el sistema se derrumba, y no existen nuevos partidos que ayuden a que se mejore esa crisis de representación, los regímenes de corte más autoritario se pueden llegar a imponer.

Este fue el caso de Venezuela. El chavismo aprovechó el colapso del sistema de partidos para cambiar las reglas de juego formales. Las nuevas que instauró, fueron en descenso de los procedimientos de representación propiamente democráticos. Ahora bien, Venezuela no es per sé una democracia en tanto la oposición aún tiene una posibilidad -aunque sea mínima- de acción en la esfera pública. Esto me lleva a retomar lo que Levitsky y Way (2004) llamaron el sistema hibrido del "autoritarismo competitivo".

En los regímenes autoritarios competitivos las instituciones democráticas formales son ampliamente vistas como medios principales para obtener y ejercer la autoridad política. Los funcionarios violan estas reglas con mucha frecuencia, hasta el punto de que el régimen no logra tener los estándares mínimos convencionales para la democracia [...] Aunque los funcionarios en los regímenes 
autoritarios competitivos puedan manipular frecuentemente las normas democráticas formales, no pueden eliminarlas o reducirlas a una mera fachada. En vez de violar abiertamente las normas democráticas (por ejemplo prohibiendo y reprimiendo a la oposición y a los medios), los funcionarios prefieren utilizar el soborno, la cooptación y otras formas más sutiles de persecución, como el uso de las autoridades de impuestos, judiciales condescendientes u otros organismos del Estado para acosar, perseguir o presionar "legalmente" un comportamiento cooperativo de los críticos (Levitsky y Way, 2004, págs. 161-163).

El régimen venezolano puede ser entendido desde este concepto, en la medida en que lo que ha tratado de hacer Chávez, es precisamente cambiar las reglas formales para que todas sus acciones -aunque sean poco democráticas- puedan estar en el marco de la legalidad. Pasaré así, a demostrar cómo ha actuado Chávez para instaurar este tipo de régimen, según los preceptos de los autores antes nombrados. Estas arenas son: la electoral, la legislativa, la judicial y los medios.

\section{Arena electoral}

Los regímenes autoritarios competitivos se caracterizan por elecciones que siempre son muy luchadas. El papel del gobierno en este caso tiene que ver más con la información parcializada sobre las elecciones y la persecución o persuasión de la oposición, de manera informal. No se presenta como tal fraude electoral, en la medida en que los votos son contabilizados de la manera correcta, pero se trata, a partir de otros medios de generar una inmolación de la oposición.

En lo que va corrido del periodo de Hugo Chávez se han presentado varias elecciones a nivel ejecutivo, legislativo y plebiscitario². En 1998 Chávez asume la presidencia con el $56.2 \%$ de los votos válidos, mientras el candidato de la oposición obtiene el $40 \%$ de los mismos (Tanaka, 2008). Chávez utiliza de excusa la nueva constitución política, que aprueba que el periodo presidencial se extienda de cuatro años a seis, para llamar a nuevas elecciones en el 2001 -con menos de dos años de haber sido elegido como presidente. En la nueva postulación gana con el $59.6 \%$ de los votos, mientras su opositor logra casi el 37,2\% de los

2 Los plebiscitos o referendos sólo se tomarán en cuanta cuando estén encaminados a realizar un cambio a nivel electoral. 
mismos. Para el 2006 la cosa no es distinta: Hugo Chávez alcanza el 62.4\% de los votos, en tanto Manuel Rosales consigue el 36.9\% (Dirección de estadísticas electorales, Consejo Supremo Electoral de Venezuela). Es imprescindible anotar que esto demuestra una oposición que no es pequeña. Al contrario, casi en todas las elecciones en las que ha participado Chávez los votos de su contrincante oscilan entre un 35 y $40 \%$, cifras que poseen un peso importante. Las últimas elecciones, celebradas en octubre de 2012, constituyen el punto culmine de la contienda electoral en Venezuela. Hugo Chávez vuelve a ganar con el 55,08\% de los votos y su opositor Henrique Capriles obtiene 44,30\%. Esto demuestra la gran polarización electoral y el alto índice de competencia en la lucha por el primer cargo de la nación, donde el último logra formar un frente de oposición entre 16 agrupaciones (Dirección de estadísticas electorales, Consejo Supremo Electoral de Venezuela).

Empero, como se puede observar, éste primer mandatario ha podido, sin salirse de la legalidad, interpretar la nueva constitución acomodándola a su favor para poder permanecer en el poder. Esto es de suprema importancia para la consideración de Venezuela como un sistema no democrático, teniendo en cuenta que la democracia necesita, en su definición mínima, la alternación del poder y la libertad de debate público (Dahl, 1992).

\section{Arena legislativa}

Un autoritarismo competitivo se caracteriza por no poseer un buen sistema de pesos y contrapesos, debido a que un poder logra imponerse sobre otros (LevitskyyWay, 2004). Hugo Chávez disolvió en el 2000 la Asamblea Nacional, que hasta el momento era bicameral; por medio de un plebiscito Venezuela decidió tener un congreso unicameral. Chávez llamó a nuevas elecciones y el AD perdió su mayoría en el parlamento: pasó de contar con 91 congresistas en las elecciones de 1998 a 30 en las elecciones del 2000. Al contrario, el MVR pasó de ocupar 67 a 78 curules en los años respectivos, convirtiéndose en el grupo más fuerte. Para el 2005 la oposición a Hugo Chávez decidió no presentarse a elecciones objetando que no tenían las garantías necesarias: 346 aspirantes a la Asamblea Nacional presentaron su renuncia a las elecciones, igual que 37 del parlamento latinoamericano y 20 del parlamento andino. Ello hizo que Chávez tuviera mayoría casi total en el legislativo, con 141 de 165 candidatos simpatizantes (Dirección de Estadísticas electorales. Consejo Nacional Electoral de Venezuela). Al observar su fracaso los partidos oponentes se volvieron 
a presentar para las elecciones del 2010 y lograron que el oficialismo perdiera la mayoría obtenida en las elecciones anteriores. Para ese año la oposición ganó 62 de los 165 escaños, lo que se consideró un logro considerable (Gámez, 2010).

Chávez posee dos medios institucionales para poder tener una alta discrecionalidad en el manejo de sus políticas. Estas son, la utilización de medios directos de participación -como plebiscitos y referendos-y la cooptación del legislativo. Como lo que muestran los números anteriores, hasta el 2010 la oposición en el parlamento era mínimo. Esta institución, pienso, constituye el espacio en el que la oposición puede tener real injerencia política y atributos de representación. Una vez la oposición se extingue en los parlamentos y el partido del ejecutivo queda con mayoría casi absoluta, se le da poder que excede los procesos democráticos y puede llegar a regímenes de cortes más autoritarios. En el caso de Venezuela la coalición oficialista ha logrado destruir las instituciones existentes y construir formas hegemónicas nuevas. El cambio institucional limita el "pluralismo y debilita la independencia y autonomía de los otros poderes del Estado” (Tanaka, 2006).

\section{Arena judicial}

Se dice que Venezuela tiene un poder judicial colonizado por el ejecutivo. El presidente ha podido violar constantemente el principio democrático de la separación de poderes, recurriendo a las instancias directas - plebiscitos- como forma de acción política. Chávez tuvo la oportunidad de cambiar la constitución y acomodarla como mejor le convenía, esto hace que pueda "eludir" de manera clara la ley, en cuanto encontró la manera de cambiarla en sí misma.

La prepotencia presidencial en la promulgación de leyes inconsultas y antidemocráticas en $2009^{3}$, cuyos contenidos habían sido rechazados en el referendo constitucional de 2007, terminó echando por tierra toda invocación constitucional chavista a la "democracia participativa y protagónica”. Si a ello agregamos la persecución y criminalización de la protesta y disidencia políticas los caminos quedaron cerrados a todo reencuentro o esfuerzo colectivo de reconciliación social (Ramos, 2011: 82).

3 Entre las más relevantes están la Ley Orgánica de Educación (LOE), la Ley Orgánica de Procesos Electorales (LOPE), la Ley Orgánica del Trabajo (LOT), la Ley Orgánica de la Fuerza Armada Nacional Bolivariana (LOFANB) y Ley Orgánica para la Ordenación y Gestión del Territorio (LOOGT). 
Como se puede observar, además de la Constitución, Hugo Chávez ha logrado sacar todo tipo de leyes para hacer válidas sus pretensiones. El sistema judicial, de hecho, no tiene capacidad de acción. Es muy difícil declararlas "inconstitucionales" en la medida en que su carta magna está diseñada precisamente para darle mayor poder al ejecutivo. En 1999, envió "la Carta del Teniente Coronel Hugo Chávez a la Corte Suprema de Justicia”, que destaca de la necesidad del cambio en el sistema de justicia. En esta medida, con el cambio de ordenamiento jurídico- constitucional, la corte se debe declarar muchas veces "incompetente", pues no hay una jurisdicción clara que le permita tomar mejores medidas (Coronel, 2006).

\section{Los medios}

Los medios de comunicación son un punto de contingencia de los regímenes autoritarios competitivos, en cuanto la mayoría son propiedad del Estado y los privados normalmente son sujetos a censuras y leyes que restringen la libertad completa de expresión y prensa (Levitsky y Way, 2004).

En la Venezuela de Hugo Chávez esta ha sido una arena completamente vulnerada. La libertad de expresión socavada mediante varias medidas del gobierno: extensión del alcance de las leyes de insulto, que castigan las actitudes irrespetuosas hacia el gobierno con penalidades más duras, lo que genera una auto-censura de los periodistas. De la misma manera, extendió y endureció el castigo de provisiones de incitación, que se encuentran definidas muy vagamente. Restringió el acceso público a información oficial y abuso el control del estado sobre las frecuencias de emisión para discriminar contra los programas demasiado críticos.

Al principio de su gobierno había un debate público muy vívido: medios pro-gobierno y anti-gobierno se expresaban virulentamente causando un ambiente de clara polarización, que se materializó en el intento de golpe de estado 2002 por parte de Pedro Caramona $^{4}$ (HRW, 2008).

A partir de ese intento, los medios de comunicación privados tomaron una posición hostil en contra de Chávez, cuestión que este aprovechó para retomar el equilibrio a su

4 Presidente de la Federación de Cámaras y Asociaciones de Comercio y Producción de Venezuela. 
favor: impuso leyes de insulto, que llevan a que los medios independientes sufran de discriminación y de censura. Todo esto ha hecho que los espacios para el debate público sean muy restringidos. Chávez alega que esto no es así, dado que ha implementado políticas que ayudan a reforzar la pluralidad y la diversidad, mediante los medios de comunicación comunitarios. Plantea que sus leyes de medio tienen por objeto empoderar al pueblo y quitarle el poder a las élites económicas, históricamente dueñas de los mismos. A pesar de su defensa por la pluralidad de voces, se advierte que no las políticas de comunicación comunitaria solo se le permiten, efectivamente, a quienes alientan el régimen (HRW, 2008). De esta manera se observa que es este campo uno de los más afectados dentro del autoritarismo competitivo chavista y, además, uno de los más importantes en términos del acceso a distintos tipos de información diferentes a las oficialistas.

\section{Reflexiones finales}

Este artículo ha tratado de ser crítico con el régimen chavista respecto a la crisis de representación partidaria. Sin embargo, es importante reconocer que los partidos políticos son solo una de las formas de representación política existentes. Entender el chavismo como fenómeno populista responde, en la misma lógica, a una forma de accountability que la ciudadanía venezolana realiza con su obsoleto bipartidismo. El populismo chavista logra permanecer en el tiempo debido a su respuesta, más o menos efectiva, a las demandas sociales.

Entender el populismo como una "forma especifica de competir por, y ejercer el poder politico" (Weyland, 2010), implica desmitificar la idea de un manejo maniqueo del líder de su electorado, y comprender a las "masas" votantes como actores racionales que encuentran en el gobierno de corte populista una posibilidad de participación más amplia que en los partidos tradicionales.

A pesar de ello, y entendiendo esto como un ejercicio académico, la caracterización del actual gobierno venezolano como un autoritarismo competitivo, radica en la idea de comprender que la forma particular de hacer política de Hugo Chávez, ha llevado a socavar el sistema de pesos y contrapesos. Un sistema de partidos colapsado no cuenta con arraigo en la sociedad, con legitimidad de sus prácticas electorales, ni con una estructura de organización institucional fuerte. Esto hace que pierda su primacía como vehículo de representación.

A falta de partidos políticos fuertes y legítimos que hicieran oposición, Chávez tuvo la oportunidad de plantear cierto tipo de reformas del diseño institucional venezolano 
que tocaron, como trató de mostrarse, todos los campos de la vida política: los sistemas electorales, los poderes judicial y legislativo, y los medios de comunicación. A partir de esto se acentúa una crisis de representación que lleva a que Venezuela haga un proceso de transición que retrocede de un modelo democrático a un régimen autoritario competitivo. Lo que se materializa en la perpetuación de su gobierno, la cooptación del congreso, la poca injerencia del poder legal y la censura constante a los medios de oposición.

\section{Bibliografía}

AGULLÓ, Juan (2009). “La forja del chavismo”. En: Estudios Latinoamericanos, núm. 24, julio-diciembre,pp. 89-113. México: Universidad Nacional Autónoma de México, Centro de Estudios Latinoamericanos (CELA) - Facultad de Ciencias Políticas y Sociales.

CORONEL, Gustavo (2006). "Corruption, Mismanagement, and Abuse of Power in Hugo Chávez's Venezuela”.En: Development Policy Analysis, noviembre, núm. 2. Washington D.C.: Center for Global Liberty and Prosperity, Cato institute. Consultado desde http://www.cato.org/pubs/dpa/dpa2.pdf

CORRALES, Javier (27 de julio de 2010). "The Venezuelan political regime today: strengths and weaknesses". En: Democracia en América Latina. Consultado el 24 de noviembre de 2011, desde: http://democraciaenamericalatina.org/articulos/javiercorrales/thevenezuelan.pdf

DAHL, Robert (1992). La democracia y sus críticos. Barcelona: Paidós.

DE LA TORRE, Carlos (2004). "Redentores populistas en el Neoliberalismo: nuevos y viejos populismos latinoamericanos". En: Revista Española de Ciencia Política, num 4.

DIETZ, Henry y David Myers (2002). “El proceso del colapso de sistemas de partidos: una comparación entre Perú y Venezuela". En: Revista Cuadernos del CENDES, vol.19, núm.50, pp. 1-33. Caracas: Universidad Central de Venezuela

CONSEJO NACIONAL ELECTORAL DE VENEZUELA (s.f.). Elecciones presidenciales cuadro comparativo 1958-2000. Dirección de estadísticas electorales, consejo supremo electoral de Venezuela. Consultado desde http:/www.cne.gov.ve/web/documentos/estadi sticas/e006.pdf

(s.f.). Elecciones 30 de Julio de 2000. Total de representantes por agrupaciones políticas y entidad. Dirección de estadísticas electorales, consejo supremo electoral de Venezuela. Consultado desde http://www.cne.gov.ve/web/documentos/ estadisticas/e041.pdf 
(s.f.). Divulgación presidencial 2012. Dirección de estadísticas electorales, consejo supremo electoral de Venezuela. Consultado desdehttp://www.cne.gob.ve/ resultado_presidencial_2012/r/1/reg_000000.html

GÁMEZ, Pablo (2010). Elecciones Venezuela: 2010. 26 de septiembre, Radio Nederland. Consultado desde http://www.rnw.nl/espanol/article/elecciones-en-venezuelaoposicion-gana-terreno

GAMUS G., Raquel (2006). El entorno regional como legitimador de los proyectos politicos. El caso venezolano. Puerto Rico: Congreso Latin American Studies Asosiation (LASA)

HAWKINS, Kirk (2007). La organización populista. Los círculos bolivarianos en Venezuela. Congreso Latinoamericano y Caribeño de Ciencias Sociales. Quito: FLACSO.

$$
\text { - (2010). Venezuela's Chavismo and populism in comparative perspective. }
$$

Cambrige: Cambrige University Press.

HUMAN RIGHT WATCH - HRW (2008). A Decade Under Chávez. Political Intolerance and Lost Opportunities for Advancing Human Rights in Venezuela. New York. Consultado desde http://www.hrw.org/reports/2008/09/18/decade-under-ch-vez

LANDER, Edgardo (2007). "El Tribunal Disciplinario del Partido Socialista Unido de Venezuela (PSUV) y la construcción de la democracia”. En: Rebelión. Consultado desde http://www.rebelion.org/noticias/2007/9/56117.pdf

LEVITSKY, Steveny Katrina Burguess (2003). "Explaining Populist Party Adaptation in Latin America: Environmental and Organizational Determinants of Party Change in Argentina, Mexico, Perú, and Venezuela". En: Comparative Political Studies, Vol. 36 No. 8, Octubre 2003, pp. 859-880. Londres: Sage Publications. Consultado desde http:// scholar.harvard.edu/levitsky/files/SL_explaining.pdf

LEVITSKY, Steven y Lucan A. Way (2004). "Elecciones sin democracia: El surgimiento del autoritarismo competitivo". En: Estudios Políticos, no. 24, enero-junio, pp. 159-176. Medellín. Universidad de Antioquia. Instituto de Estudios Políticos. Consultado desde http:// biblioteca.clacso.edu.ar/ar/libros/colombia/iep/24/8\%20autoritarismo\%20competitivo.pdf

MAINWARING, Scott y Timothy R. Scully (1995). "La institucionalización de los sistemas de partidos en América Latina” en Revista de Ciencia Política, vol. XVII, No. 1-2, pp.63-101.

NOHLEN, Dieter (2004) Sistemas electorales y partidos políticos. México D.F.: Fondo de Cultura Económica. 
PARTIDO SOCIALISTA UNIDO DE VENEZUELA (s.f). “Tribunal disciplinario del PSUV”. Consultado desde http://www.psuv.org.ve/tribunal-disciplinario/

RAMOS, Alfredo (2011). "La "revolución" que no fue. Desgobierno y autoritarismo en la Venezuela de Chávez". En: Estudios Políticos, núm. 38, pp. 69-91. Instituto de Estudios Políticos, Universidad de Antioquia. Consultado desdehttp://aprendeenlinea.udea.edu. co/revistas/index.php/estudiospoliticos/article/viewFile/10016/9233

RIVAS, José Antonio (2000). "La vulnerabilidad de la democracia y el rediseño institucional en Venezuela". En: Foro Internacional, núm. 162, pp. 718-742. Centro de Estudios Internacionales del Colegio de México.

TANAKA, Martin (2006). Los sistemas de partidos en los países andinos, 19802005: reformismo institucional, autoritarismos competitivos y los desafíos actuales. Working Paper, núm. 324. Indiana, EE.UU.: Helen Kellogg Institute for International Studies de la Universidad de Notre Dame. Consultado desdehttp://www.nd.edu/ kellogg/ publications/workingpapers/WPS/324.pdf

- (2008). "De la crisis al derrumbe de los sistemas de partidos, y los dilemas de la representación democrática: Perú y Venezuela”. En: Mainwaring, Scott, Ana María Bejarano y Eduardo Pizarro, eds., La crisis de representación democrática en los países andinos, pp. 89-131. Bogotá: Norma.

WEYLAND, Kurt (2001). "Clarifying a Contested Concept Populism in the Study of Latin American Politics”. En: Comparative Politics, num 34. 